\title{
Aprendendo História através de Museus Virtuais: uma parceria entre Professores e Museólogos
}

\author{
Eline F. de A. Welter'1, Luciana A. M. Zaina², Andréia D. de Leles³, Monique S. \\ Ribeiro $^{2}$ \\ ${ }^{1}$ Departamento de Computação - IFSP - Campus Itapetininga \\ Av. João O. de Oliveira, 1561, Vl. Asem, CEP 18202-000 - Itapetininga - SP - Brasil \\ ${ }^{2}$ Departamento de Computação - UFSCAR - Campus Sorocaba \\ Rod. João L. dos Santos, Km 110 - SP-264, CEP 18052-780 - Sorocaba - SP - Brasil \\ ${ }^{3}$ Value Projetos, Treinamentos e Consultoria \\ Rua Maria do Carmo B. Cortez, 116, sala 1, Parque 3 meninos - Sorocaba - SP - Brasil \\ \{eliwelter, monisribeiro\}@gmail.com, lzaina@ufscar.br, \\ andreia@valueprojects.com.br
}

\begin{abstract}
This article reports an experience on the use of virtual museums through computers and mobile devices to support the learning and teaching of History in the elementary school. The experiment was performed with three schools in the region of Sorocaba, two of them are public and one is private, using a tool called e-Museum. The results demonstrated the potential of the tool and the feasibility of teaching through virtual museums.
\end{abstract}

Resumo. Este artigo apresenta o relato de experiência sobre o uso de museus virtuais através de computadores e pequenos dispositivos como apoio ao ensino de História em séries do Ensino Fundamental II. A experiência foi realizada com três escolas na região de Sorocaba, sendo duas delas estaduais e uma privada, utilizando uma ferramenta denominada e-Museu. A análise dos resultados demonstrou o potencial da ferramenta e a viabilidade do ensino através dos museus virtuais.

\section{Introdução}

Os museus podem ser considerados um grande repositório de expressão artística e histórica, contribuindo fortemente com a memória da humanidade e com o incentivo à educação e cultura, através da conservação das obras e realização de eventos expositivos. Por relatar a história em diferentes temáticas, o museu pode ser considerado um instrumento de estudo em diversas modalidades de educação, sendo essa uma das premissas que justifique sua grande importância na educação e cultura. Portanto, podese concluir que os museus têm significativa participação no processo educativo e firmamento na formação de cidadãos, conforme relatado nos trabalhos de Bianconi e Vieira (2007); Berry, Sheard e Quartly (2011) e Sarah, Prihatmanto e Rusmin (2012). Oliveira (2013) reporta estudos e iniciativas mundiais de parcerias entre escolas e museus. 
O cenário brasileiro, entretanto apresentam dados que reportam o não acesso aos museus. De acordo com dados de uma pesquisa realizada pelo IBGE - Instituto Brasileiro de Geografia e Estatística em 2009, apresentada no site oficial do IBRAM ${ }^{1}$, apenas 6\% dos brasileiros já adentraram um museu. Conforme site do IBRAM, o Brasil possui 2.970 museus, espalhados, em sua maioria, em grandes centros das regiões sul, sudeste e litoral, dificultando as visitações de outras localizações, de maneira a restringir acesso à história e memória nacional, que poderiam colaborar diretamente com a cultura e formas de ensino-aprendizagem [IBRAM 2009]. No outro extremo está o crescente desenvolvimento tecnológico com novos meios de acesso à Internet. Um grande desafio que a área de educação tem buscado transpor é unir a crescente imersão dos jovens na tecnologia e seu uso como apoio ao desenvolvimento da aprendizagem e cultura. $\mathrm{O}$ crescente uso de dispositivos móveis (smartphones e tablets) é apontado por Nielsen Online (2012) em uma pesquisa realizada entre julho de 2011 e julho de 2012 em diversos países, incluindo o Brasil. A pesquisa aponta que o tempo que uma pessoa passa acessando a Web (seja via aplicativo ou via browser) via dispositivo móvel cresceu 21\% no período. Além disto, o uso de Web Mobile ${ }^{2}$ e de aplicativos mobile, cresceu em torno de $80 \%$ em um ano. Enquanto que no mesmo período o uso do PC caiu 4\%. O tempo que uma pessoa gasta em aplicações mobile cresceu $120 \%$ no período.

Von Staa (2009) apresenta em um estudo feito com escolas públicas e privadas que a experiência educacional utilizando computadores móveis é muito positiva e motivante. Já Velloso e Pintomarinho (2010) apontam que a escola, por sua vez, ainda não assumiu a interatividade e a multifuncionalidade da web. Está evidente que enquanto recurso para a aprendizagem, o uso do computador e da Internet não é significativo na escola, nem fora dela, ainda que os jovens lancem mão da web para cumprir a tradicional tarefa da pesquisa escolar. O MEC (Ministério da Educação e Cultura) vem trabalhando para tentar inserir a escola no contexto tecnológico. Há quatro anos vem trabalhando pela inserção do uso de tablets nas escolas públicas, porém relata que a falta de um conteúdo curricular digital adequado, resistência dos professores e custo elevado ainda são alguns dos impeditivos. Já no ano de 2014 o MEC promete distribuir quase 500 mil tablets [Tablet na escola, 2014]. Independente do acesso dentro dos muros escolares, existe um crescimento no uso de pequenos dispositivos como smartphones e tablets por parte dos estudantes. Os pequenos dispositivos que vem sendo lançados pela indústria já possuem recursos diversificados de interação por diferentes canais, tais como voz, gestos, caneta, movimentos, toque, etc. Porém, o que se observa é que grande parte das aplicações que são utilizadas nos dispositivos não utilizam os diferentes canais disponíveis.

O objetivo deste artigo é apresentar o relato da experiência sobre o uso de museus virtuais através de computadores e pequenos dispositivos (tablets $\mathrm{e}$ smartphones) como apoio ao ensino de História em séries do Ensino Fundamental II. A experiência foi realizada com três escolas na região de Sorocaba, sendo duas delas estaduais e uma privada, utilizando uma ferramenta denominada e-Museu, disponível para computadores e pequenos dispositivos. A iniciativa faz parte do projeto "Uma

\footnotetext{
${ }^{1}$ IBRAM - Instituto Brasileiro de Museus

${ }^{2}$ Mobile - são dispositivos móveis como celular, smartphone ou tablets.
} 
Linha de Produtos de Software para Intensificar o Desenvolvimento de Museus Virtuais com Interação Multimodal" (processo no 2013/25572-7) patrocinado pela FAPESP ${ }^{3}$ com parceria da Secretaria da Cultura e Lazer da cidade de Sorocaba ${ }^{4}$. O projeto tem por objetivo intensificar o desenvolvimento de museus virtuais para o entendimento dos fatos históricos com o propósito de prover a parceria entre a cultura e a educação. Durante a experiência foram coletados e posteriormente analisados dados relacionados à aceitação da ferramenta proposta e aos hábitos tecnológicos dos alunos participantes.

O restante do artigo está organizado em: Seção 2 que aborda a questão dos museus virtuais e apresenta a ferramenta e-Museu; Seção 3 apresenta a metodologia de condução da experiência; Seção 4 discute os resultados; e por fim a Seção 5 realiza as considerações finais, apontando os trabalhos futuros.

\section{Museus Virtuais}

Ao visitar um museu, o visitante pode entender fatos históricos e relacioná-los com a atualidade, desenvolvendo experiências significativas, caracterizadas como ensinoaprendizagem informal. Porém, a carga informacional que pode ser apresentada ao visitante do museu virtual pode causar problemas na atenção e entendimento do real objeto foco da visita. Marty e Twidale (2004) e Karoulis, Sylaiou e White (2006) relatam que, normalmente, os museus virtuais apresentam uma excessiva quantidade de informações ou apresentam interfaces com usuário que chama mais atenção do que o objeto de estudo, o que faz com que o usuário acabe se desviando de seu real objetivo. Considerando as deficiências apontadas pelos trabalhos anteriores, analisou-se os sites de três grandes museus conhecidos mundialmente: museu do Louvre ${ }^{5}$, The Virtual Egyptian Museum ${ }^{6}$ e British Museum ${ }^{7}$ [Universia Brasil, 2012], com o propósito de identificar aspectos de interface e interação multimodal. Observou-se se os mesmos tinham i. interface diferenciada para dispositivos móveis, podendo ser via browser ou aplicativo; e ii. canais de interação diferenciados (voz, gesto ou movimento). Apenas o British Museum atendeu o item i. e nenhum dos museus analisados atendeu o item ii.

Considerando os estudos realizados foi desenvolvida a ferramenta e-Museu que explora canais de interação que os museus analisados não apresentam. O e-Museu é um subproduto do projeto "Uma Linha de Produtos de Software para Intensificar o Desenvolvimento de Museus Virtuais com Interação Multimodal", com o propósito de flexibilizar o uso de museus virtuais para aprendizagem. A ferramenta foi validada com 12 docentes do Ensino Fundamental através da técnica de grupos em foco com protótipos de baixa fidelidade [Preece; Rogers;Sharp, 2013] em uma oficina realizada em setembro de 2013. A partir da validação foi desenvolvida uma versão funcional. Embora existam diversos museus virtuais, o e-Museu permite que o professor e/ou o museólogo construa a sala de visitação, de acordo com o tema que deseja abordar. A interação do professor e/ou museólogo possui acesso via computador e os alunos podem acessar via navegador web ou aplicativo no dispositivo móvel (versão atual disponível

\footnotetext{
${ }^{3}$ http://www.fapesp.br

${ }^{4}$ http://www.sorocaba-sp.com.br/1/1269/municipais/secretaria-da-cultura-e-lazer-de-sorocaba.html

${ }^{5} \mathrm{http}: / /$ www.louvre.fr

${ }^{6} \mathrm{http}: / /$ www.virtual-egyptian-museum.org

${ }^{7} \mathrm{http}: / /$ www.britishmuseum.org
} 
para Android $^{8}$ ). Em ambas versões (via navegador ou dispositivo móvel) é necessário ter acesso a Internet, pois o repositório encontra-se em um servidor na web. Na tela de entrada o visitante escolhe a galeria que deseja visualizar (A). Durante a visitação o aluno tem acesso a imagens, textos e um agente de voz (narrador) que estão em consonância com o tema abordado (B). O aluno pode optar, por exemplo, em não utilizar o agente de voz. Além disto, é disponibilizado um quiz ${ }^{9}$, com questões objetivas (C), no qual o aluno pode testar seus conhecimentos, enquanto navega pelo e-Museu (Figura 1).

\section{Contexto e Metodologia}

Durante o mês de maio de 2014 foi realizado o experimento em três escolas da região de Sorocaba, sendo as escolas estaduais EE Prof. Júlio Bierrenbach Lima e EE Prof. Modesto Tavares de Lima e o colégio COC Santa Rosália uma instituição privada. O ambiente físico utilizado foi o laboratório de informática de cada escola com acesso à Internet por banda larga, e os 174 participantes eram alunos pertencentes ao $6^{\circ}$ e $7^{\circ}$ anos do Ensino Fundamental II (com idades entre 10 e 14 anos), provenientes de diferentes classes sociais e com realidades de aprendizagem diversificadas. Foram envolvidos no experimento três professores de História (um de cada instituição participante), um Museólogo da Secretaria da Cultura de Sorocaba, seis pesquisadores de interação humano-computador (dois professores, três mestrandos e uma aluna de iniciação científica). $\mathrm{O}$ tema histórico do experimento foi o Tropeirismo e foram utilizadas imagens do Museu Histórico Sorocabano, cedidas pela Secretaria da Cultura parceira.

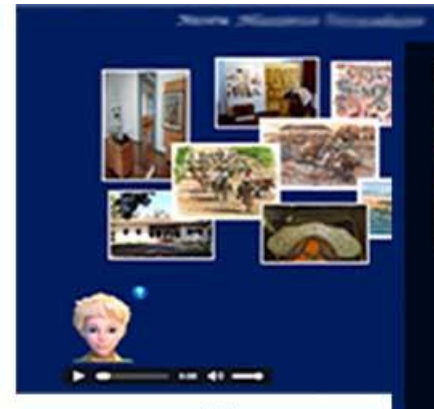

(A)

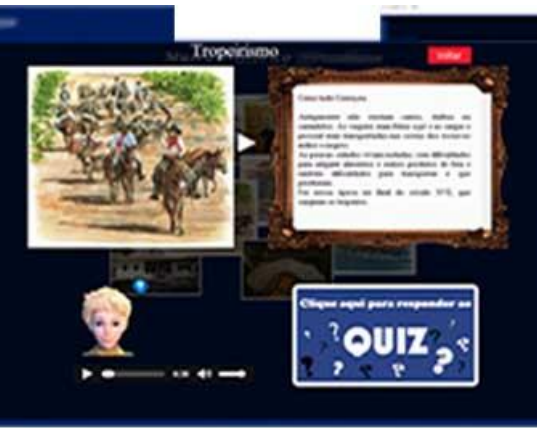

(B)

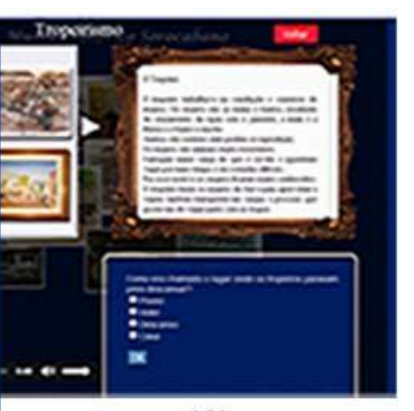

(C)

Figura 1. Interface do aluno e-Museu

Para o experimento foram definidos os atores e suas respectivas participações em cada etapa (Tabela 1). Os atores primários $(\mathrm{P})$ eram aqueles responsáveis por produzir os resultados e agiam diretamente na etapa e os secundários (S) possuíam ações indiretas, podendo intervir com opiniões sobre conteúdos e objetos, ou mesmo realizar perguntas relativas ao funcionamento do experimento. Antes de iniciar as fases do experimento, uma das pesquisadoras de interação apresentou aos docentes das escolas envolvidas o tema do experimento (que havia sido proposto pela Secretaria da Cultura parceira) e a ferramenta e-Museu, discutindo as diversas formas de interação.

\footnotetext{
${ }^{8} \mathrm{http}: / /$ www.android.com/

${ }^{9}$ Quiz - termo em inglês utilizado para avaliar pessoas com um questionário.
} 
Os professores deram um retorno muito positivo sobre o tema e a ferramenta e se propuseram a participar ativamente do experimento.

Durante a fase de Planejamento, museólogo, professores e pesquisadores interagiram com o objetivo de construir a sala de visitação e seus artefatos. $O$ formulário de observação visava identificar as escolhas feitas pelos alunos participantes, relativo aos objetos de interação e seu comportamento em relação ao museu proposto. $\mathrm{O}$ formulário de feedback dos participantes tinha por objetivo coletar dados sobre uso da Internet e de pequenos dispositivos, sua aceitação em relação ao e-Museu e aos objetos de interação disponíveis. Os formulários foram elaborados por dois pesquisadores de interação.

Tabela 1. Etapas do Experimento e seus Atores

\begin{tabular}{|c|c|c|c|c|c|}
\hline \multirow{2}{*}{\multicolumn{2}{|c|}{ Etapas }} & \multicolumn{4}{|c|}{ Atores } \\
\hline & & \multirow{2}{*}{$\frac{\text { Professor }}{\mathrm{P}}$} & \multirow{2}{*}{$\begin{array}{c}\text { Museólogo } \\
\mathrm{S} \\
\end{array}$} & \multirow{2}{*}{$\frac{\text { Aluno }}{-}$} & \multirow{2}{*}{$\begin{array}{c}\text { Pesquisador } \\
\mathrm{S} \\
\end{array}$} \\
\hline 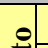 & Elaboração do conteúdo textual e voz e do quiz. & & & & \\
\hline : & $\begin{array}{l}\text { Escolhas das imagens do museu em consonância } \\
\text { com o tema. }\end{array}$ & $\mathrm{s}$ & $P$ & - & $\mathrm{S}$ \\
\hline$\frac{\pi}{2}$ & Elaboração da visitação no e-Museu. & $\mathrm{P}$ & $\mathrm{S}$ & - & $\mathrm{P}$ \\
\hline$\frac{\bar{\pi}}{\underline{a}}$ & $\begin{array}{l}\text { Elaboração dos formulários de observação e de } \\
\text { feedback dos participantes. }\end{array}$ & - & - & - & $\mathrm{P}$ \\
\hline \multirow{5}{*}{ 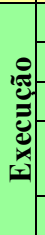 } & Explanação pré-experimento aos participantes. & $\mathrm{S}$ & - & $S$ & $\mathrm{P}$ \\
\hline & Visitação ao e-Museu. & - & - & $\mathrm{P}$ & - \\
\hline & Observação das ações dos participantes. & $\mathrm{S}$ & - & - & $\mathrm{P}$ \\
\hline & $\begin{array}{l}\text { Responder questionário de feedback sobre o e- } \\
\text { Museu. }\end{array}$ & - & - & $P$ & - \\
\hline & Entrevista com o professor. & $\mathrm{P}$ & - & - & $\mathrm{S}$ \\
\hline 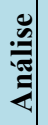 & Consolidação dos dados coletados. & $S$ & $S$ & - & $\mathrm{P}$ \\
\hline
\end{tabular}

$\mathrm{Na}$ Execução, inicialmente um pesquisador de interação e o professor de História realizavam a explanação sobre o que era o experimento, porém sem explicar como utilizar a ferramenta e-Museu. Também questionavam os alunos sobre o conhecimento da existência física do museu e sobre o que conheciam a respeito do tema tropeirismo. Os alunos foram separados em grupos de 12 alunos por sessão para facilitar a observação. A cada sessão era em média, seis alunos utilizando navegador em computadores (dois observadores) e seis utilizando dispositivos móveis (três observadores). Optou-se por um número maior de observadores para os dispositivos móveis, pois a observação da interação necessita ser mais minuciosa. Enquanto os alunos realizavam o experimento, os observadores relatavam no formulário de observação: algum tipo de dificuldade em realizar alguma tarefa, dificuldade em interagir com a interface, preferências pelas mídias disponíveis e grau de satisfação demonstrado pelo aluno. Assim que os alunos finalizavam a visitação eles tinham alguns minutos para navegação livre no e-Museu. A Figura 2 apresenta os alunos interagindo com diferentes dispositivos. Ao finalizar a visitação e a navegação livre os alunos respondiam ao questionário de feedback de forma anônima. Os docentes participantes foram entrevistados para se obter o feedback sobre a experiência pela perspectiva deles. Ao final, os condutores do experimento (professor e pesquisador de interação) questionavam os alunos novamente sobre o tema Tropeirismo para verificar nas respostas se haviam entendido o tema proposto. 
A análise ocorreu após o término do experimento em todas as escolas e resultou em um volume grande de dados e possibilidades de análises. Para este artigo serão apresentados, na próxima seção, alguns dados e análises realizadas.

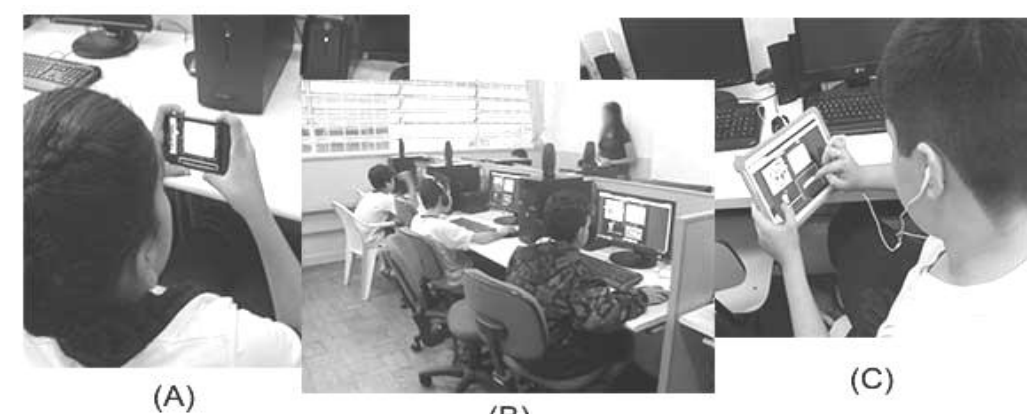

(B)

Figura 2. Alunos realizando o experimento em diferentes dispositivos.

\section{Resultados e Discussão}

Neste artigo serão apresentados resultados identificados via observação e via feedback respondidos por museólogo, docentes e alunos. O foco é apresentar dois conjuntos de resultados: "hábitos sobre uso da Internet e de dispositivos" e "aceitação do uso do eMuseu"; sendo que o segundo conjunto de resultados inclui-se também os dados do museólogo e dos professores.

Durante a fase de Planejamento observou-se que houve um alinhamento sobre o objetivo pedagógico envolvido entre os professores e museólogo, resultando em olhares complementares: i. museólogo - preocupação sobre o objeto do museu que seria utilizado como ilustração e que demonstrasse de fato uma evidência histórica; ii. professores - elaboração de conteúdos completos e sucintos, pois a ideia era que os alunos explorassem o objeto do museu. Todos indicaram que o e-Museu é um ambiente excelente e adequado para apoiar as aulas.

A distribuição do número de alunos participantes (total de 174) na Execução do experimento foi: 88 da Escola 1 (EE Prof. Júlio Bierrenbach Lima), 37 da Escola 2 (EE Prof. Modesto Tavares de Lima) e 49 da Escola 3 (COC Santa Rosália), sendo respectivamente as duas primeiras alunos do $6^{\circ}$ ano de escolas estaduais e a última alunos do $7^{\circ}$ ano de um colégio particular. Do total de participantes, 95 (55\%) utilizaram o e-Museu em pequenos dispositivos: 44 (50\%) da Escola 1, 16 (43\%) da Escola 2 e 35 (71\%) da Escola 3. Para algumas sessões na Escola 1 e 3, foi dada a opção de escolha sobre qual dispositivo o aluno gostaria de usar. A preferência foi pelos dispositivos móveis. Os alunos que escolheram o computador justificaram que se sentiam mais familiarizados em utilizá-lo e alguns reportaram que gostam do dispositivo móvel apenas para jogos.

Considerando os resultados sobre "hábitos sobre uso da Internet e de dispositivos" foram estabelecidos três critérios para análise de frequências: $\mathrm{C} 1 \leftarrow$ participantes que acessam a Internet em casa ou na casa de parentes e amigos de 4 a 7 dias por semana; $\mathrm{C} 2 \leftarrow \mathrm{C} 1$ e acesso através de dispositivo móvel; $\mathrm{C} 3 \leftarrow$ realizam a atividade de pesquisas escolares na Internet. Os resultados gerais foram (Gráfico 1 - por escola): 165 (95\%) alunos do total relataram que acessam a Internet em sua casa ou na 
casa de parentes e amigos; dos 165, 122 (74\%) atenderam o critério $\mathrm{C} 1$; dentre os 122 , 67 (55\%) foram identificados no critério C2; e dentre os 174 participantes 94 (54\%) atenderam o critério $\mathrm{C} 3$.

Gráfico 1. Hábitos sobre uso da Internet e dispositivos por Escola

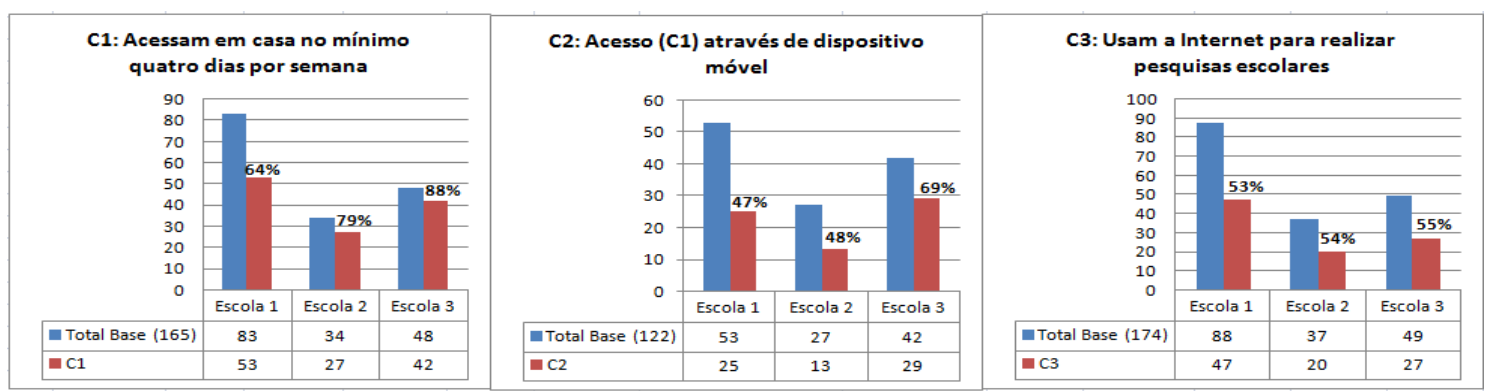

Fonte: Dados da pesquisa

O resultado de $74 \%$ dos alunos que acessam a Internet no mínimo 4 vezes por semana é superior a média geral de acesso por alunos do ensino fundamental para a região Sudeste $(51 \%)$ revelada em pesquisa realizada pelo Centro de Estudos sobre as Tecnologias da Informação e da Comunicação [CETIC.br, 2012], apresentando uma tendência de crescimento durante os anos. Porém, o resultado de $74 \%$ está próximo aos $75 \%$ que a mesma pesquisa indica quando considera que os alunos acessam a Internet em casa. A maioria dos alunos (95\%) afirmou que faz uso da Internet em casa ou na casa de parentes e amigos, sendo que o meio de acesso mais utilizado ainda é o computador. Porém, observa-se que na Escola 3, que é um colégio privado, os dispositivos móveis são maioria. Quando considerado o acesso via celular (ou smartphone), independente do local de acesso, chega-se ao total de $41 \%$ entre as escolas, valor muito próximo aos $43 \%$ de acessos via celular apresentado na pesquisa [CETIC.br, 2012]. Este resultado demonstra que há um crescimento significativo no uso de dispositivos móveis pelos participantes, mesmo em escolas estaduais quando há presença de alunos com renda mais baixa. Contudo, fica evidente que embora os participantes acessem com frequência a Internet não realizam tal tarefa na escola. Outro ponto, é que a atividade de uso para "pesquisas escolares", marcada por 94 participantes, não é a atividade mais realizada pelos mesmos quando estão na Internet, conforme pode ser observado no Gráfico 2.

\section{Gráfico 2. Atividades mais realizadas pelos participantes na Internet}

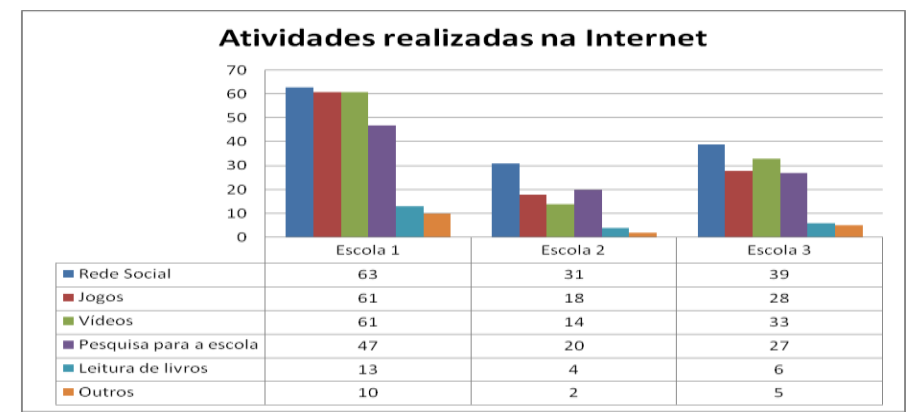

Fonte: Dados da pesquisa

Para análise da coleta de feedback dos alunos sobre a "aceitação do uso do eMuseu" foram adotados quatro critérios: $\mathrm{C} 4 \leftarrow$ aceitação do uso do e-Museu; C5 $\leftarrow$ recursos de interação que mais gostou (podia selecionar mais do que um); $\mathrm{C} 6 \leftarrow$ dificuldades dos alunos durante a interação; $\mathrm{C} 7 \leftarrow$ entusiasmo do aluno com o experimento. Os 
resultados dos dois últimos critérios foram extraídos da coleta dos observadores. O Gráfico 3 apresenta os resultados de C4 e C5 por escola. Considerando os 174 participantes, 129 (74\%) dos alunos classificaram o e-Museu como "muito legal", 42 (24\%) como "legal" e apenas $3(2 \%)$ apontaram não ter gostado (C4). Observou-se que os alunos que "não gostaram" não indicaram em questão posterior sobre os recursos que mais gostou a opção "não gostei de nada". Notou-se que os três alunos utilizaram o smartphone como meio de acesso, isto alertou os pesquisadores de interação como um sinalizador para readequação dos elementos de interação para este dispositivo. Para os recursos de interação disponíveis (C5) a opção que mais apareceu foi "gostei de tudo" com 95 marcações (55\%) feitas de forma única (53 - 60\% da Escola 1, 19 - 51\% da Escola 2 e 23 - 47\% da Escola 3); logo depois aparecem as "imagens" e o "quiz"; não houve nenhuma marcação para a opção "não gostei de nada". A escolha do recurso "imagens" foi um resultado importante, pois os professores de história relataram que os alunos, usualmente, possuem dificultades na interpretação de imagens no contexto histórico. Outro ponto importante, foi que houveram mais marcações no recurso "texto" do que no "narrador", demonstrando a preferência por leitura.

Gráfico 3. Aceitação e recursos que mais gostou do e-Museu

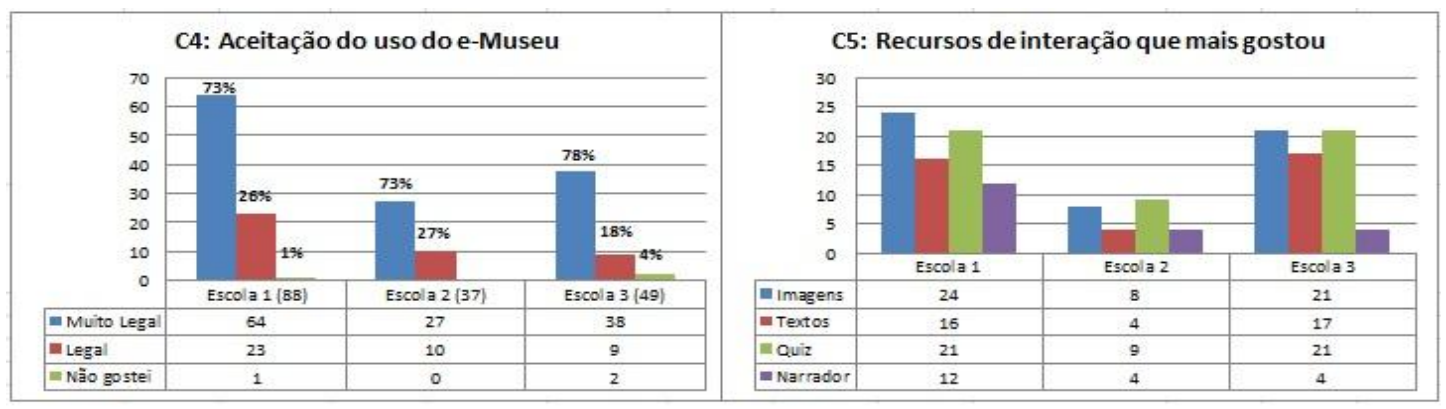

Fonte: Dados da pesquisa

Através da observação foi possível extrair alguns dados e análises. Durante o experimento o professor e o pesquisador de interação não ensinaram o aluno como usar o e-Museu, pois um dos objetivos era verificar a facilidade de uso. Dos 174 participantes, 141 (81\%) "não necessitaram de nenhuma ajuda" para utilizar o e-Museu (C6), distribuídos em 66 (75\%) da Escola 1, 28 (76\%) da Escola 2 e 47 (96\%) da Escola 3 , demonstrando que a interação da ferramenta era de fácil aprendizado. Dentre os alunos que tiveram dificuldades, estas aconteceram no quiz e foram: i. rótulos que não estavam claros e ii. uso dos botões de rádio para selecionar a alternativa correta da questão. A dificuldade ii. ocorreu apenas para os alunos que utilizavam smartphones, devido ao tamanho dos botões. Tais observações demonstraram a necessidade de rever os elementos de interação do quiz. Também foi observado o entusiasmo do aluno com a participação no experimento, através de duas perspectivas: i. sua atenção e satisfação durante e depois de finalizar o experimento e ii. sua reação no tempo que tinha disponível para navegação livre. Relacionado a i. (C6) dos 174 participantes, 155 (89\%) demonstraram atenção e satisfação com o experimento, distribuídos em 82 (93\%) da Escola 1, 28 (76\%) da Escola 2 e 45 (92\%) da Escola 3, o que permite inferir que os alunos gostaram não só da ferramenta, mas também da forma como o conteúdo foi abordado. Além disto, ao final das sessões diversos alunos questionavam se teriam acesso ao link da ferramenta para poderem acessar de casa e se haveria outros temas de história estudados através da ferramenta. Considerando ii. (C7), apenas 12 alunos (7\%) 
aguardaram a finalização do experimento sem realizar nenhuma outra interação. Destes, apenas $3(2 \%)$ deles demonstraram a "não atenção e satisfação" durante a visitação e durante o tempo livre. Outra observação relevante é que durante o período de navegação livre foi relatado que quase $70 \%$ dos alunos refizeram o quiz (duas ou mais vezes), apontando que o aluno sentiu-se motivado para acertar o máximo de questões possíveis. Os alunos que não refizeram o quiz foram os que acertaram todas as questões na primeira tentativa; estes exploravam de uma maneira geral, olhando as imagens, texto e ouvindo o narrador de forma livre. Ao finalizar todas as etapas do experimento os professores e pesquisadores de interação questionavam novamente os alunos sobre o que era o Tropeirismo e os alunos demonstravam satisfação respondendo detalhes do que foi estudado durante a visitação.

Durante a entrevista com os docentes e museólogo, todos relataram que gostaram da experiência e que repetiriam com outros conteúdos. Um dos pontos reportados pelos docentes foi que gostariam que fosse possível na ferramenta e-Museu associar os fatos históricos do passado ao presente. Também sugeriram alterações para a interface do e-Museu, como por exemplo, possibilidade de alterar a organização dos elementos de interação, a moldura dos objetos e adição de uma linha do tempo para observação de fatos históricos.

\section{Considerações Finais}

Este artigo apresentou o relato de experiência sobre o uso de museus virtuais através de computadores e pequenos dispositivos (tablets e smartphones) como apoio ao ensino de História em séries do Ensino Fundamental II. O experimento motivou todos os participantes, sendo que alguns se manifestaram para perguntar onde era o museu e se poderiam ir com suas famílias visita-lo, outros perguntavam se podiamos passar o site ou aplicativo para que eles pudessem acessar de suas residências, fortalecendo o propósito de despertar interesse e aproximar o aluno do museu. Também foi possível observar que os hábitos não são diferentes quando analisados alunos de escola públicas e de escola particular.

Um dos trabalhos futuros é aprofundar as análises dos dados coletados, incluindo análises separadas para pequenos dispositivos e computador. O cruzamento dos dados sobre os hábitos e os dados observados também se constitui em resultados importantes. Outros dados sobre quais os recursos a mais os alunos gostariam que tivesse no e-Museu necessitam ser analisados. Outro trabalho futuro se constitui no desenvolvimento de novas funcionalidades na visão dos alunos, professores $\mathrm{e}$ museólogos. Para os alunos deseja-se incorporar outras opções de interação como movimentos para pequenos dispositivos e busca por voz; integração do museu virtual com as redes sociais.

\section{Agradecimentos}

Agradecemos à Secretaria da Cultura e Lazer de Sorocaba, o Museu Histórico Sorocabano, às escolas EE Prof. Júlio Bierrenbach de Lima e Colégio COC Santa Rosália de Sorocaba e EE Prof Modesto Tavares de Lima de Itapetininga pela parceria no experimento. Também agradecemos a FAPESP pelo apoio financeiro ao projeto. 


\section{Referências}

Berry, G.; Sheard, J.; Quartly, M. (2011) “A Virtual Museum of Computing History: an educational resource bringing the relationship between people and computers to life". Proceeding of $13^{\text {th }}$ Australasian Computer Education.Conference, Australia.

Bianconi, M.L.; Vieira, V. A. (2007) "Importância do Museu Nacional da UFRJ para o Ensino Não-Formal de Ciências”. Revista Ciências \& Cognição, Vol. 1, pp. 21-36.

Cetic.br (2012) Centro de Estudos Sobre as Tecnologias da Informação e Comunicação (Brasil). Pesquisas e Indicadores: Tic na Educação. 2012. Disponível em: http://www.cetic.br/educacao/2012/alunos/ index.html>. Acessado em: 20/06/2014.

IBRAM (2009) Brasil, Ministério da Cultura. "Portal de Comunicação: 94\% dos brasileiros nunca visitaram um museu". Disponível em: http://www.clicrbs.com.br/ especial/rs/portal-social/19,0,3054406,94-dos-brasileiros-nunca-visitaram-ummuseu.html. Acessado em 02/05/2013.

Karoulis, A.; Sylaiou, S.; White, M. (2006) "Usability Evaluation of a Virtual Museum Interface”, INFORMATICA, Vol. 17, N. 3, pp. 363-380.

Marty, P.; Twidale, M. (2004) "Lost in gallery space: a conceptual framework for analyzing the usability flaws of museum web sites". First Monday, Vol. 9, N. 9.

Nielsen Online (2012) "Social media report 2012: social media comes of age", Disponível em: http://www.nielsen.com/us/en/newswire/2012/social-media-report2012-social-media-comes-of-age.html, Acessado em: 02/07/2013.

Oliveira, Ge. (2013) "O museu como um instrumento de reflexão social”, MIDAS , Vol. $2,2013$.

Preece, J.; Rogers, Y.;Sharp, H. (2013) "Interaction Design: Beyond Human-Computer Interaction", Willey.

Sarah, L.L.; Prihatmanto, A.S.; Rusmin, P.H. (2012) "The design and implementation discovery learning method on virtual museum of Indonesia: A case study museum of geology for rock materials". Proceedings of 2012 International Conference on Digital Object, pp. $1-5$.

Tablet na escola (2014) "Governo impulsiona uso de tablet na escola", Disponível em: http://www.todospelaeducacao.org.br/educacao-na-midia/indice/29338/governoimpulsiona-uso-de-tablet-na-escola/, Acessado em: 02/07/2014.

Universia Brasil (2012) "46 museus virtuais para você visitar de graça”, Disponível http://noticias.universia.com.br/destaque/noticia/2012/02/16/912114/46-museusvirtuais-voce-visitar-graca.html, Acessado em 01/09/2014.

Velloso, M. J. M.; Pintomarinho, S. P. (2010) “O Uso do Computador e da Internet por Estudantes de 11 a 14 anos de uma escola municipal". Anais do WIE, Belo Horizonte, pp. 1324 - 1333.

Von Staa, B. (2009) "Computadores móveis na escola: reação de pais, alunos e professores", Anais do SBIE 2009. 